PROCEEDINGS OF THE

AMERICAN MATHEMATICAL SOCIETY

Volume 126, Number 2, February 1998, Pages 383-389

S 0002-9939(98)04478-5

\title{
THE EXPLICIT SOLUTION OF A DIFFUSION EQUATION WITH SINGULARITY
}

\author{
MICHIAKI WATANABE AND SHUJI WATANABE \\ (Communicated by Palle E. T. Jorgensen)
}

\begin{abstract}
We give the explicit solution of the Cauchy problem for the diffusion equation with a singular term:

$$
\begin{gathered}
(\partial / \partial t) u=(\partial / \partial x)^{2} u-\left(k / x^{2}\right) u, \quad t>0, \quad x \in \mathbf{R}^{1} ; \\
u(0, x)=f(x), \quad x \in \mathbf{R}^{1},
\end{gathered}
$$

where $k>-1 / 4$. We construct the solution on the basis of a generalization of the Fourier transform. We next show that the solution is expressed by an analytic semigroup, and examine smoothness of $x \mapsto u(t, x)$ and continuity of $x \mapsto u(t, x) / x^{\beta}(\beta>0)$.
\end{abstract}

\section{The MAIn RESUlT}

In this paper we give the explicit solution of the Cauchy problem for the diffusion equation with a singular term:

$$
\begin{gathered}
\frac{\partial u}{\partial t}=\frac{\partial^{2} u}{\partial x^{2}}-\frac{k}{x^{2}} u, \quad t>0, \quad x \in \mathbf{R}^{1} ; \\
u(0, x)=f(x), \quad x \in \mathbf{R}^{1},
\end{gathered}
$$

where $k>-1 / 4$. We next show that the solution is expressed by an analytic semigroup, and examine smoothness of $x \mapsto u(t, x)$ and continuity of $x \mapsto u(t, x) / x^{\beta}$ $(\beta>0)$.

Let $f_{+}(x)=\{f(x)+f(-x)\} / 2, f_{-}(x)=\{f(x)-f(-x)\} / 2$, and let $I_{\nu}$ be a modified Bessel function, for which we refer the reader to the books $[\mathrm{E}]$ and $[\mathrm{OK}]$. Our main result is the following.

Theorem 1.1. Let $f \in L^{2}\left(\mathbf{R}^{1}\right)$. Then the explicit solution of the Cauchy problem (1.1), (1.2) is given by

$$
\begin{aligned}
u(t, x)=\int_{\mathbf{R}^{1}} \frac{\sqrt{|x \xi|}}{4 t} e^{-\left(x^{2}+\xi^{2}\right) /(4 t)}\left\{I_{a-1 / 2}\left(\frac{|x \xi|}{2 t}\right) f_{+}(\xi)\right. & \\
& \left.+\operatorname{sgn}(x \xi) I_{b+1 / 2}\left(\frac{|x \xi|}{2 t}\right) f_{-}(\xi)\right\} d \xi
\end{aligned}
$$

Received by the editors May 7, 1996.

1991 Mathematics Subject Classification. Primary 35K15, 35K22; Secondary 42A38.

Key words and phrases. Diffusion equation with singularity, generalized Fourier transform, analytic semigroup.

The second author was partially supported by Grant-in-Aid for Scientific Research (No.07740175), Ministry of Education, Science, Sports and Culture. 
for a.a. $x \in \mathbf{R}^{1}$, where $a, b>-1 / 2$ and

$$
a(a-1)=b(b+1)=k .
$$

Remark. When $k=0,(1.1)$ is reduced to the usual diffusion equation. Indeed, setting $a=b=0$ in the explicit solution above and noting the formulas $I_{-1 / 2}(z)=$ $\sqrt{2 /(\pi z)} \cosh (z)$ and $I_{1 / 2}(z)=\sqrt{2 /(\pi z)} \sinh (z)$, we obtain for a.a. $x \in \mathbf{R}^{1}$,

$$
u(t, x)=\int_{\mathbf{R}^{1}} \frac{1}{\sqrt{4 \pi t}} e^{-\left(x^{2}+\xi^{2}\right) /(4 t)}\left\{\cosh \left(\frac{x \xi}{2 t}\right) f_{+}(\xi)+\sinh \left(\frac{x \xi}{2 t}\right) f_{-}(\xi)\right\} d \xi
$$

and hence arrive at the well-known formula represented by the Gaussian kernel:

$$
u(t, x)=\frac{1}{\sqrt{4 \pi t}} \int_{\mathbf{R}^{1}} e^{-(x-\xi)^{2} /(4 t)} f(\xi) d \xi .
$$

In what follows we therefore confine ourselves to the case where $k>-1 / 4$ and $k \neq 0$.

\section{Construction of the solution}

In this section, using a generalization of the Fourier transform, we construct the explicit solution of (1.1), (1.2) in the case where $k>-1 / 4$ (and $k \neq 0$ ) and give the proof of Theorem 1.1. The classical formula (1.3) for $k=0$ is derived, for example, by means of the Fourier transform. Our generalized transform $B_{c} \quad(c>-1 / 2)$ has been introduced by Ohnuki and S. Watanabe [OW, Theorems 3.2 and 3.4] as a unitary operator from $L^{2}\left(\mathbf{R}^{1}\right)$ onto itself. We outline it for the reader's convenience. We have

$$
\begin{aligned}
& B_{c} u(y)=\underset{L \rightarrow \infty}{\operatorname{li} \text { m. }} \int_{-L}^{L} \overline{\varphi_{c}(x y)} u(x) d x, \\
& B_{c}^{*} u(x)=\underset{L \rightarrow \infty}{\operatorname{li} \text { i.m. }} \int_{-L}^{L} \varphi_{c}(x y) u(y) d y
\end{aligned}
$$

for $u \in L^{2}\left(\mathbf{R}^{1}\right)$. Here the function $\varphi_{c}$ given by

$$
\varphi_{c}(x y)=\frac{\sqrt{|x y|}}{2}\left\{J_{c-1 / 2}(|x y|)+i \operatorname{sgn}(x y) J_{c+1 / 2}(|x y|)\right\}, \quad c>-\frac{1}{2},
$$

was obtained by Ohnuki and Kamefuchi [OK, pp. 289-296], where $J_{\nu}$ is a Bessel function. If $c=0$, then $\varphi_{c=0}(x y)=e^{i x y} / \sqrt{2 \pi}$. In this case $B_{c}$ coincides with the Fourier transform, and so it can be regarded as a generalization of the Fourier transform.

For each $m=0,1,2, \ldots$, let

$$
\mathcal{H}_{c}^{m}\left(\mathbf{R}^{1}\right)=\left\{u \in L^{2}\left(\mathbf{R}^{1}\right): \int_{\mathbf{R}^{1}}\left(1+y^{2}\right)^{m}\left|B_{c} u(y)\right|^{2} d y<\infty\right\}, \quad c>-\frac{1}{2} .
$$

The space of Sobolev type $\mathcal{H}_{c}^{m}\left(\mathbf{R}^{1}\right)$ is a Hilbert space with inner product

$$
(u, v)_{m}=\int_{\mathbf{R}^{1}}\left(1+y^{2}\right)^{m} B_{c} u(y) \overline{B_{c} v(y)} d y
$$


and norm $|u|_{m}=(u, u)_{m}^{1 / 2}$ [W2] (see also [W1]). Furthermore, in [W2, Lemma $2.4]$ it is shown that the operator $\left(-i \mathcal{D}_{c}\right)^{m}$ is self-adjoint on the set $\mathcal{H}_{c}^{m}\left(\mathbf{R}^{1}\right)$, where

$$
\mathcal{D}_{c}=\frac{d}{d x}-\frac{c}{x} R, \quad R u(x)=u(-x), \quad c>-\frac{1}{2} .
$$

See also [WW, Theorem 1] and [OW, Theorem 2.1 and Proposition 4.2] for the self-adjointness of $-i \mathcal{D}_{c}$. This operator $\mathcal{D}_{c}$ appears in a one-dimensional harmonic oscillator governed by Wigner's commutation relations [Wi], and the expression (2.4) was derived by Yang [Y]. As is well known, multiplication by $y$ is self-adjont on the set $D(y)=\left\{u(y): u, y u \in L^{2}\left(\mathbf{R}^{1}\right)\right\}$. With the aid of the transform $B_{c}$, the operators $\left(-i \mathcal{D}_{c}\right)^{m}$ and $y^{m}$ are unitarily equivalent to each other [OW, Proposition 4.2]:

$$
B_{c}\left(-i \mathcal{D}_{c}\right)^{m} B_{c}^{*}=y^{m}, \quad B_{c} \mathcal{H}_{c}^{m}\left(\mathbf{R}^{1}\right)=D\left(y^{m}\right) .
$$

For unitary equivalence, see e.g. Goldstein [G, pp. 94-95].

Remark. The transform $B_{c}$ depends on the constant $c$, and so does $\mathcal{H}_{c}^{m}\left(\mathbf{R}^{1}\right)$. When $c=0, B_{c=0}$ coincides with the Fourier transform and $\mathcal{H}_{c=0}^{m}\left(\mathbf{R}^{1}\right)$ turns out to be the usual Sobolev space $H^{m}\left(\mathbf{R}^{1}\right)$.

We now turn to the Cauchy problem (1.1), (1.2). Let us decompose $u(t, x)$ into the sum of the even part $u_{+}(t, x)$ and the odd part $u_{-}(t, x)$ :

$$
u(t, x)=u_{+}(t, x)+u_{-}(t, x),
$$

where

$$
u_{ \pm}(t, x)=\frac{1}{2}\{u(t, x) \pm R u(t, x)\}=\frac{1}{2}\{u(t, x) \pm u(t,-x)\} .
$$

Then the problem (1.1), (1.2) is decomposed into

$$
\begin{array}{ll}
\frac{\partial u_{+}}{\partial t}=\left(\frac{\partial^{2}}{\partial x^{2}}-\frac{k}{x^{2}}\right) u_{+}, & u_{+}(0, x)=f_{+}(x) ; \\
\frac{\partial u_{-}}{\partial t}=\left(\frac{\partial^{2}}{\partial x^{2}}-\frac{k}{x^{2}}\right) u_{-}, & u_{-}(0, x)=f_{-}(x) .
\end{array}
$$

Using the equality $\mathcal{D}_{c}^{2}=(\partial / \partial x)^{2}-x^{-2} c(c-R)$, we can rewrite $(2.6)$ and $(2.7)$ as

$$
\begin{aligned}
\frac{d u_{+}}{d t}=\mathcal{D}_{a}^{2} u_{+}, & u_{+}(0)=f_{+} ; \\
\frac{d u_{-}}{d t}=\mathcal{D}_{b}^{2} u_{-}, & u_{-}(0)=f_{-},
\end{aligned}
$$

where $a, b>-1 / 2, a(a-1)=b(b+1)=k$ and $f \in L^{2}\left(\mathbf{R}^{1}\right)$. In order to solve (2.8) and (2.9), we have only to deal with

$$
\frac{d u}{d t}=\mathcal{D}_{c}^{2} u, \quad u(0)=f \in L^{2}\left(\mathbf{R}^{1}\right), \quad c>-\frac{1}{2},
$$

and then to discuss the even and odd parts of the solution $u$.

By (2.5), the transform $B_{c}$ turns (2.10) into

$$
\frac{d U}{d t}=-y^{2} U, \quad U(0)=B_{c} f
$$


where $U=B_{c} u$. An elementary calculation gives $U(t, y)=e^{-t y^{2}} B_{c} f(y) . \quad U(t, \cdot)$ evidently belongs to $D\left(y^{m}\right)$ for every positive integer $m$. Hence (2.5) implies that $u(t, \cdot)$ belongs to $\mathcal{H}_{c}^{m}\left(\mathbf{R}^{1}\right)$ (see also (2.3)). Thus, recalling (2.1), we have

$$
u(t, x)=\underset{L \rightarrow \infty}{\lim } \int_{-L}^{L} \varphi_{c}(x y) e^{-t y^{2}} B_{c} f(y) d y .
$$

Since $e^{-t y^{2}} B_{c} f \in D(y)$, it follows from [OW, Lemma 3.3] that

$$
u(t, x)=v(t, x) \quad\left(\text { a.a. } x \in \mathbf{R}^{1}\right), \quad \text { where } v(t, x)=\int_{\mathbf{R}^{1}} \varphi_{c}(x y) e^{-t y^{2}} B_{c} f(y) d y .
$$

Lemma 2.1. Let $v$ be given by (2.11). Then

$$
v(t, x)=\int_{\mathbf{R}^{1}} f(\xi)\left\{\int_{\mathbf{R}^{1}} \varphi_{c}(x y) \overline{\varphi_{c}(\xi y)} e^{-t y^{2}} d y\right\} d \xi .
$$

Proof. Set $F_{L}(y)=\int_{-L}^{L} \overline{\varphi_{c}(\xi y)} f(\xi) d \xi$. Then, letting $L \rightarrow \infty$ gives

$$
\begin{aligned}
& \left|v(t, x)-\int_{\mathbf{R}^{1}} \varphi_{c}(x y) e^{-t y^{2}} F_{L}(y) d y\right| \\
& \quad \leq \sqrt{\int_{\mathbf{R}^{1}}\left|\varphi_{c}(x y)\right|^{2} e^{-2 t y^{2}} d y}\left\|B_{c} f-F_{L}\right\| \rightarrow 0,
\end{aligned}
$$

where $\|\cdot\|$ denotes the norm of $L^{2}\left(\mathbf{R}^{1}\right)$. Fubini's theorem therefore implies

$$
v(t, x)=\lim _{L \rightarrow \infty} \int_{-L}^{L} f(\xi)\left\{\int_{\mathbf{R}^{1}} \varphi_{c}(x y) \overline{\varphi_{c}(\xi y)} e^{-t y^{2}} d y\right\} d \xi,
$$

which proves the lemma.

It remains to compute the integral

$$
I(x, \xi)=\int_{\mathbf{R}^{1}} \varphi_{c}(x y) \overline{\varphi_{c}(\xi y)} e^{-t y^{2}} d y .
$$

\section{Lemma 2.2.}

$$
I(x, \xi)=\frac{\sqrt{|x \xi|}}{4 t} e^{-\left(x^{2}+\xi^{2}\right) /(4 t)}\left\{I_{c-1 / 2}\left(\frac{|x \xi|}{2 t}\right)+\operatorname{sgn}(x \xi) I_{c+1 / 2}\left(\frac{|x \xi|}{2 t}\right)\right\} .
$$

Proof. Recalling (2.2), we obtain

$$
\begin{aligned}
I(x, \xi)= & \frac{\sqrt{|x \xi|}}{2} \int_{0}^{\infty} e^{-t y^{2}} y J_{c-1 / 2}(|x| y) J_{c-1 / 2}(|\xi| y) d y \\
& +\operatorname{sgn}(x \xi) \frac{\sqrt{|x \xi|}}{2} \int_{0}^{\infty} e^{-t y^{2}} y J_{c+1 / 2}(|x| y) J_{c+1 / 2}(|\xi| y) d y
\end{aligned}
$$

An appeal to the formula [E, (23), p. 51] ( for Re $\nu>-1$ and $|\arg a|<\pi / 4$ )

$$
\int_{0}^{\infty} e^{-a^{2} y^{2}} y J_{\nu}(p y) J_{\nu}(q y) d y=\frac{1}{2 a^{2}} e^{-\left(p^{2}+q^{2}\right) /\left(4 a^{2}\right)} I_{\nu}\left(\frac{p q}{2 a^{2}}\right)
$$

completes the proof. 
From Lemmas 2.1 and 2.2, we thus find that the solution $u$ of (2.10) is given by

$$
\begin{aligned}
u(t, x)=\int_{\mathbf{R}^{1}} \frac{\sqrt{|x \xi|}}{4 t} e^{-\left(x^{2}+\xi^{2}\right) /(4 t)}\left\{I_{c-1 / 2}\left(\frac{|x \xi|}{2 t}\right)\right. & \\
& \left.+\operatorname{sgn}(x \xi) I_{c+1 / 2}\left(\frac{|x \xi|}{2 t}\right)\right\} f(\xi) d \xi
\end{aligned}
$$

for a.a. $x \in \mathbf{R}^{1}$. Recalling (2.8) and (2.9), we see that Theorem 1.1 is true.

Remark. If $-1 / 4<k<3 / 4$ and $k \neq 0$, then $a=-b, b+1$. Setting $a=-b$, $a=b+1$ in (2.8), we have the following two equations:

$$
\frac{d u_{+}}{d t}=\mathcal{D}_{-b}^{2} u_{+}, \quad \frac{d u_{+}}{d t}=\mathcal{D}_{b+1}^{2} u_{+} .
$$

\section{Properties of the SOlution}

In this section we show that each of $u_{ \pm}(t, \cdot)$ in Theorem 1.1 is expressed by an analytic semigroup, and examine smoothness of $x \mapsto u_{ \pm}(t, x)$ and continuity of $x \mapsto u_{ \pm}(t, x) / x^{\beta} \quad(\beta>0)$. To begin with we try to construct the solution of (1.1), (1.2) by the formula $u_{ \pm}(t, x)=\exp \left(t \mathcal{D}_{c}^{2}\right) f_{ \pm}(x)$ based on the abstract theory of semigroup generation:

Lemma 3.1. The operator $\mathcal{D}_{c}^{2}$ generates an analytic semigroup $\left\{\exp \left(t \mathcal{D}_{c}^{2}\right): t>0\right\}$ on $L^{2}\left(\mathbf{R}^{1}\right)$.

Proof. By (2.5) we have $B_{c} \mathcal{D}_{c}^{2} B_{c}^{*}=-y^{2}$. The multiplication operator $-y^{2}$ generates the semigroup $\left\{\exp \left(-t y^{2}\right): t>0\right\}$, which is evidently an analytic semigroup. Since the transform $B_{c}$ is unitary, the result follows.

From this lemma we obtain that the solution of (1.1), (1.2) with $f \in L^{2}\left(\mathbf{R}^{1}\right)$ is expressed by $u_{ \pm}(t, x)=\exp \left(t \mathcal{D}_{c}^{2}\right) f_{ \pm}(x)$, and $u_{ \pm}(t, \cdot)$ belongs to $\mathcal{H}_{c}^{2 m}\left(\mathbf{R}^{1}\right)$ for every $m=1,2, \ldots$ and $t>0$. Combining the inclusion $\exp \left(t \mathcal{D}_{c}^{2}\right) L^{2}\left(\mathbf{R}^{1}\right) \subset$ $\mathcal{H}_{c}^{2 m}\left(\mathbf{R}^{1}\right)$ and an embedding theorem of Sobolev type on $\mathcal{H}_{c}^{2 m}\left(\mathbf{R}^{1}\right)$ (which was recently established by $\mathrm{S}$. Watanabe [W2, Theorem 1.2]), we can study further properties of $u_{ \pm}(t, \cdot)$. To this end we introduce the following spaces. For each $m=0,1,2, \ldots$, let

$$
\begin{aligned}
& \mathcal{B}^{m}\left(\mathbf{R}^{1}\right)=\left\{u \in C^{m}\left(\mathbf{R}^{1}\right): \frac{d^{k} u}{d x^{k}}(k=0,1, \ldots, m) \text { are bounded on } \mathbf{R}^{1}\right\} \\
& \mathcal{F}^{m}\left(\mathbf{R}^{1}\right)=\left\{u(x): u, \frac{u}{x^{k}}(k=1,2, \ldots, m) \text { are continuous and bounded on } \mathbf{R}^{1}\right\} .
\end{aligned}
$$

As is well known, $\mathcal{B}^{m}\left(\mathbf{R}^{1}\right)$ is a Banach space with norm

$$
|u|_{\mathcal{B}^{m}}=\max _{k=0,1, \ldots, m}\left\{\sup _{x \in \mathbf{R}^{1}}\left|\frac{d^{k} u}{d x^{k}}(x)\right|\right\},
$$

and $\mathcal{F}^{m}\left(\mathbf{R}^{1}\right)$ is a Banach space with norm

$$
|u|_{\mathcal{F} m}=\max _{k=0,1, \ldots, m}\left\{\sup _{x \in \mathbf{R}^{1}}\left|\frac{u(x)}{x^{k}}\right|\right\},
$$

as is shown in [W2, Lemma 2.1]. We describe below the statement of an embedding theorem of Sobolev type for the operator $\mathcal{D}_{c}$. It reveals the smoothness of $u \in$ $\mathcal{H}_{c}^{m}\left(\mathbf{R}^{1}\right)$ and continuity of $x \mapsto u(x) / x^{\beta} \quad(\beta>0)$. 
Lemma 3.2 ([W2, Theorem 1.2]). Suppose $c>1$. Then, for each $u \in \mathcal{H}_{c}^{m}\left(\mathbf{R}^{1}\right)$, there is an element $v \in \mathcal{B}^{\alpha}\left(\mathbf{R}^{1}\right) \cap \mathcal{F}^{\beta}\left(\mathbf{R}^{1}\right)$ such that $u(x)=v(x) \quad\left(\right.$ a.a. $\left.x \in \mathbf{R}^{1}\right)$, i.e.,

$$
\mathcal{H}_{c}^{m}\left(\mathbf{R}^{1}\right) \subset \mathcal{B}^{\alpha}\left(\mathbf{R}^{1}\right) \cap \mathcal{F}^{\beta}\left(\mathbf{R}^{1}\right)
$$

where

$$
\begin{aligned}
& \alpha= \begin{cases}m-1 & (c=2 n), \\
\min (m-1, c-1) & (c=2 n+1), \\
\min (m-1,[c]) & (\text { otherwise }),\end{cases} \\
& \beta= \begin{cases}\min (m-1, c) & (c=2 n), \\
\min (m-1, c-1) & (c=2 n+1), \\
\min (m-1,[c]) & (\text { otherwise })\end{cases}
\end{aligned}
$$

with $n=1,2,3, \ldots$ Moreover, $|v|_{\mathcal{B}^{\alpha}} \leq c_{1}|u|_{m}$ and $|v|_{\mathcal{F}^{\beta}} \leq c_{2}|u|_{m}$, where $c_{1}$ and $c_{2}$ are positive constants that depend on $m$ and $c$ only.

Let $m=1,2,3, \ldots$ Then the solution $u_{+}(t, \cdot)$ in Theorem 1.1 (see also (2.8)) is in $\mathcal{H}_{a}^{2 m}\left(\mathbf{R}^{1}\right.$ ), while the solution $u_{-}(t, \cdot)$ (see also (2.9)) is in $\mathcal{H}_{b}^{2 m}\left(\mathbf{R}^{1}\right)$. We thus obtain the following result.

Theorem 3.3. (i) Suppose $a>1$. Then the even part $u_{+}(t, \cdot)$ of the solution $u$ of the problem (1.1), (1.2) belongs to $\mathcal{B}^{\alpha}\left(\mathbf{R}^{1}\right) \cap \mathcal{F}^{\beta}\left(\mathbf{R}^{1}\right)$, where

$$
\alpha=\left\{\begin{array}{ll}
\text { any positive integer } & (a=2 n), \\
a-1 & (a=2 n+1), \\
{[a]} & \text { (otherwise), }
\end{array} \quad \beta= \begin{cases}a & (a=2 n), \\
a-1 & (a=2 n+1), \\
{[a]} & \text { (otherwise) }\end{cases}\right.
$$

with $n=1,2,3, \ldots$.

(ii) Suppose $b>1$. Then the odd part $u_{-}(t, \cdot)$ belongs to $\mathcal{B}^{\alpha}\left(\mathbf{R}^{1}\right) \cap \mathcal{F}^{\beta}\left(\mathbf{R}^{1}\right)$, where

$$
\alpha=\left\{\begin{array}{ll}
\text { any positive integer } & (b=2 n), \\
b-1 & (b=2 n+1), \\
{[b]} & (\text { otherwise }),
\end{array} \quad \beta= \begin{cases}b & (b=2 n), \\
b-1 & (b=2 n+1), \\
{[b]} & (\text { otherwise })\end{cases}\right.
$$

with $n=1,2,3, \ldots$.

Remark. It is interesting that $u_{+}(t, \cdot)$ belongs to $C^{\infty}\left(\mathbf{R}^{1}\right)$ if $k=2 n(2 n-1)$, and so does $u_{-}(t, \cdot)$ if $k=2 n(2 n+1)$ for $n=1,2,3, \ldots$.

\section{REFERENCES}

[E] A. Erdélyi (ed.), Tables of integral transforms, vol. II, McGraw-Hill, New York, 1954. MR 16:468c

[G] J. A. Goldstein, Semigroups of linear operators and applications, Oxford University Press, New York, 1985 / Clarendon Press, Oxford, 1985. MR 87c:47056

[OK] Y. Ohnuki and S. Kamefuchi, Quantum field theory and parastatistics, University of Tokyo Press, Tokyo, 1982 / Springer-Verlag, Berlin, Heidelberg and New York, 1982. MR 85b:81001

[OW] Y. Ohnuki and S. Watanabe, Self-adjointness of the operators in Wigner's commutation relations, J. Math. Phys. 33 (1992), 3653-3665. MR 93h:81065

[WW] M. Watanabe and S. Watanabe, Self-adjointness of the momentum operator with a singular term, Proc. Amer. Math. Soc. 107 (1989), 999-1004. MR 90g:81035

[W1] S. Watanabe, Sobolev type theorems for an operator with singularity, Proc. Amer. Math. Soc. 125 (1997), 129-136. MR 97c:47044

[W2] S. Watanabe, An embedding theorem of Sobolev type for an operator with singularity, Proc. Amer. Math. Soc. 125 (1997), 839-848. MR 97e:46042 
[Wi] E. P. Wigner, Do the equations of motion determine the quantum mechanical commutation relations?, Phys. Rev. 77 (1950), 711-712. MR 11:706e

[Y] L. M. Yang, A note on the quantum rule of the harmonic oscillator, Phys. Rev. 84 (1951), 788-790. MR 13:804e

Faculty of Engineering, Nitgata University, Niigata 950-21, Japan

E-mail address: m.watanabe@geb.ge.niigata-u.ac.jp

Department of Mathematics, Toyota National College of Technology, Eisei-Cho 2-1, TOYOTA-SHI 471 , JAPAN

E-mail address: swtnb@tctcc.cc.toyota-ct.ac.jp 